\title{
CLINICO-CHEMICAL CONFERENCE
}

\author{
Hormones and lipid metabolism \\ Clinico-Chemical Conference held at The Royal Postgraduate Medical School, \\ arranged by DR B. LeWIS and DR G. R. THOMPSON, with \\ Professor LARS CARLSON as guest speaker
}

Prof. Booth: This morning's session is going to consider the various ways in which hormones and lipid metabolism can be related. We shall start with Dr Barry Lewis, who is going to talk about diabetes and lipid metabolism and the effect of insulin on lipid metabolism-Dr Barry Lewis.

\section{Diabetes and lipid metabolism}

DR LEWIS: There is a complex relationship between the diabetic state and lipid metabolism, and we will consider free fatty acids first and then triglycerides. Some of the relationships to be discussed are shown in Fig. 1.

\section{(a) Free fatty acids}

It is well known that there is commonly an increase in free fatty acid (FFA) concentration in plasma. In collaboration with Professor Russell Fraser, Mr M. Mattock and Dr M. Mancini, we have measured the turnover of FFA in maturity-onset diabetics before

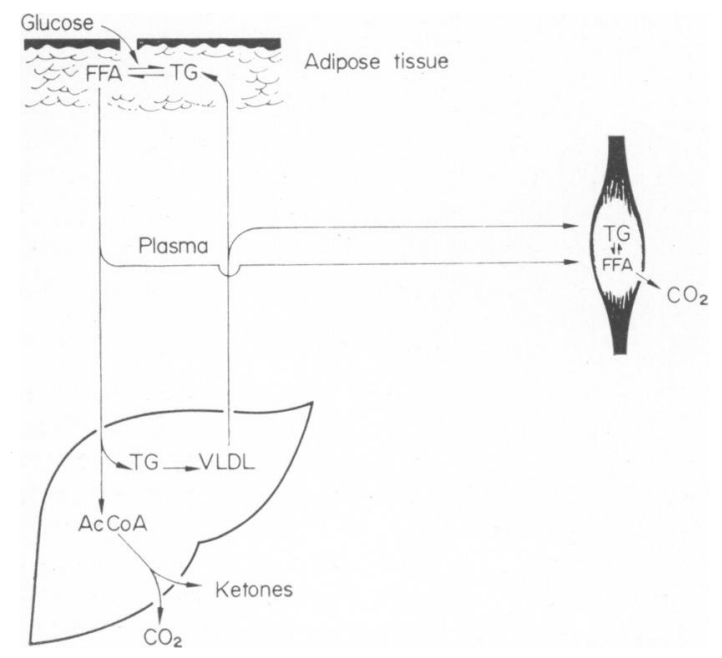

Fig. 1. Free fatty acid (FFA) and triglyceride (TG) relationships. In diabetes, free fatty acid release into plasma is increased; hepatic secretion of very lowdensity lipoprotein (VLDL) may be impaired, and uptake of triglyceride (transported as VLDL) from plasma, e.g. to adipose tissue and muscle appears to be reduced. and during treatment, by an isotope-dilution method. The normal range for FFA production from adipose tissue by this technique is of the order of 5-10 $\mu \mathrm{mol} / \mathrm{min} / \mathrm{kg}$ body weight. In all of the patients the turnover of FFA was increased in the untreated state; after 2-12 weeks of dietary treatment the rate fell towards or into the normal range. So we may infer that there was overproduction of FFA by adipose tissue and this is the reason for the high plasma FFA levels. Also we found an approximate correlation between FFA plasma concentration and production rate.

\section{(b) Triglycerides}

The second point is also familiar; in uncontrolled diabetics the triglyceride concentration in fasting plasma is sometimes high, but irrespective of the actual value, it seems to fall during treatment. This is seen both in insulin-dependent and in maturityonset diabetes. The reduction is especially striking in those patients who intitially have high levels. Even by the strictest standards of normality, only about half of our untreated diabetic patients had hypertriglyceridaemia; however, diabetics with vascular complications more often have hypertriglyceridaemia than those without vascular complications (Albrink, Lavietes \& Man, 1963). In an interesting population study, Abrams et al. (1969) have shown a strong positive correlation between the serum triglyceride level in apparently normal people and carbohydrate intolerance: the area under the glucose tolerance curve correlated well with the fasting serum triglyceride. In patients with familial endogenous hypertriglyceridaemia, too, mild diabetes is rather common; about $50 \%$ of the series of Glueck, Levy $\&$ Fredrickson (1969a) had carbohydrate intolerance. It has been suggested that insulin may play a pathogenetic role in this form of hyperlipidaemia (Reaven et al., 1967; Stout \& Vallance-Owen, 1969); but Glueck found that insulin responsiveness was as often low as high. It is quite possible that endogenous hypertriglyceridaemia is not a single metabolic entity, and that future work will define a sub-group in which hyperinsulinism is a regular feature. 
Insulin and lipid metabolism

Insulin has several effects on lipid metabolism, and the individual effects are sometimes mutually antagonistic.

\section{(a) Triglyceride removal from plasma}

Adipose tissue and muscle appear to be the most important sites of triglyceride uptake from plasma. Triglyceride circulates in the plasma in chylomicra, and prebetalipoprotein. The first stage is the splitting of this triglyceride at the capillary endothelium by lipoprotein lipase with release of glycerol back into plasma. The second stage is the intracellular reesterification of the fatty acids back to triglyceride which is then stored. The esterification process cannot re-use glycerol, but depends on the availability of glycerol-3-phosphate derived from carbohydrate metabolism; by promoting glucose utilization, and increasing glycerol-3-phosphate availability, insulin may thus facilitate the removal of triglyceride from plasma. In addition, insulin increases lipoprotein lipase activity, at least in adipose tissue, so the first stage of triglyceride uptake is also insulin-dependent.

To obtain a measure of the fractional turnover rate of triglyceride in diabetic patients, we have modified a method developed in the laboratories of Professor Lars Carlson in which the disappearance of an injected fat emulsion is measured. Before treatment, the fractional disappearance rateranged from 1 to $7 \%$ min with about half of our patients showing subnormal rates (normal range about $3 \cdot 5-10 \% / \mathrm{min}$ ); during treatment the fractional turnover rate inincreased in most cases, and so it seems that the dietary treatment of maturity-onset diabetes may accelerate triglyceride clearance. This may account at least in part for the reduction of plasma triglyceride concentration.

Now, in experimental work, insulin administration for short periods, 6-24 hr, reduces serum triglyceride concentration in normal man (Jones \& Arky, 1965), and in other species; similarly if one administers glucose to the fasted rat the triglyceride level falls, and Baker (1968), who has done this work, has kinetic data which also suggest that this fall is due to increased removal rate.

\section{(b) Triglyceride secretion into plasma}

If we now look at the rather more conflicting picture of insulin effects on the secretion of triglyceride into plasma, that is, the secretion of very low density lipoprotein (VLDL) by the liver, the sequence of events is probably this. The main precursor of plasma endogenous triglyceride is plasma FFA. FFA taken up by the liver is re-esterified to triglyceride which is coupled with apoprotein, and secreted as very low density lipoprotein. As insulin decreases free fatty acid production by adipose tissue it is likely that hepatic uptake of this precursor of triglyceride becomes diminished. Both insulin effects that I have mentioned above would tend to reduce the plasma triglyceride level.

\section{(c) Conflicting mechanisms}

However, there are mechanisms which have been demonstrated by in vitro studies with liver slices (Renold et al., 1955) and with the perfused rat liver (Haft, 1968) which indicate that insulin-lack depresses fatty acid synthesis. The liver from diabetic rats also has a decreased ability to incorporate fatty acid into triglyceride which is partly restored by insulin (van Harken et al., 1967). The same laboratory has shown that the liver of alloxan diabetic rats has an impaired ability to incorporate amino acids into lipoprotein. Anti-insulin serum decreases the incorporation of acetate into free fatty acids, hence the removal of the effect of insulin seems to impair fatty acid synthesis. And finally, Basso \& Havel (1970) have reported that in diabetic dogs, hyperlipidaemia occurs despite decreased incorporation of FFA into plasma triglyceride.

\section{(d) Triglycerides and hyperinsulinism}

Returning now to Abrams' survey, there was shown a quite significant positive correlation between the fasting serum triglyceride level and the insulin responsiveness to oral glucose in a normal population. It seems that a high insulin response can accompany a high serum triglyceride. And other possibly related effects of insulin have been shown, in retrospective studies on patients with ischaemic heart disease who are commonly glucose intolerant; their insulin responsiveness is frequently exaggerated (Tzagournis et al., 1968). To some extent, this may be explained by the co-existence of obesity with the ischaemic heart disease, and hyperinsulinism is not invariably observed (Jackson et al., 1970) in patients with ischaemic heart disease.

\section{(e) Consequences of high FFA in diabetes}

You will all be familiar with the in vitro evidence that free fatty acid inhibits some stages in carbohydrate utilization (Randle et al., 1963). Whatever the role of these effects in the mechanism of diabetes, high free fatty acid levels may largely be responsible for the ketosis of diabetes, providing a substrate for ketogenesis, and may contribute to the insulin resistance which is characteristic of diabetic ketoacidosis. When FFA is infused into experimental animals, depending on the experimental situation the blood glucose may rise or fall, but what does seem significant is that free fatty acid infusion consistently produces an increase in insulin levels (Greenough, Crispin \& Steinberg, 1967). 
In summary, insulin has conflicting effects on plasma triglyceride metabolism; in acute experiments, mechanisms tending to reduce triglyceride levels predominate, and defects in these mechanisms probably explain the hypertriglyceridaemia of diabetes. In large population groups, on the other hand, hyperinsulinism may accompany hypertriglyceridaemia but its causal role remains to be established. It is possible that at least in some patients hyperinsulinism may lead to hypertriglyceridaemia.

PRof. Booth: Well, thank you very much Dr Lewis. Shall we have questions now and then general discussion at the end? Anybody any queries they would like to bring up? I was always taught that fat burns in the flame of carbohydrate.

DR LEwIS: Well, in starvation, fatty acids tend to be oxidized or converted to ketones. As regards oxidation of fatty acids and glucose, $I$ think that there is actually a reciprocal relationship between these substrates.

PROF. BOOTH: The inference is that insulin action on fat metabolism is mediated through its effect on carbohydrate metabolism?

DR LEWIS: I think there are direct effects as well. It is a hormone with many indepent sites of action.

Prof. Booth: Right, thank you very much Dr Lewis. Now, Professor Carlson, can we ask you to tell us about catecholamines?

\section{Catecholamines and lipid metabolism}

PROF. CARLSON: I think I would like to begin where Dr Lewis ended and say that I think that we presently are in a very interesting state of development where we are beginning to understand that not only hormones have effects on lipid metabolism but that also lipids may affect hormones. This, in a way, is similar to the way we know that hyper- and hypoglycaemia may stimulate secretion of insulin or glucagon. For instance, we are now beginning to realize that lipids, for example, free fatty acids (FFA), may also have influence on the secretion of insulin.

\section{(a) Historical and general review}

I will start with reviewing the effects of catecholamines on lipid metabolism historically. We have known for quite a long time that epinephrine and norepinephrine influence lipid metabolism. In the 1920s it was found that epinephrine raised plasma lipid levels. Then it was found that epinephrine increased the total fatty acid level in plasma; more specifically in 1955 it was found that epinephrine raised triglyceride levels. Perhaps the most important contribution came in 1956 when independently Dole and Gordon \& Cherkes in the U.S. showed that epinephrine increased the levels of FFA. Later on it was also shown that the serum cholesterol may increase in response to epinephrine. Also it is known that catecholamines affect tissue lipids. In 1937 it was shown that injections of epinephrine induced fatty livers and in 1958 it was shown that fat infiltration could result also in other organs in response to injection of catecholamines (see Carlson, Boberg \& Hogstedt, 1965). In adipose tissue the historical development was as follows: it was shown in 1957 by Wadström that epinephrine injection raised the concentration of the lower glycerides, i.e. diglycerides and monoglycerides in adipose tissue, suggesting lipolysis. Later on, it was shown by in vitro studies that epinephrine increased fat mobilization from adipose tissue in the form of FFA, and more recently that epinephrine increases the levels of cyclic AMP in adipose tissue.

So, if we summarize the available evidence, we can say that catecholamines increase triglyceride levels in most tissues and that blood lipid fractions, as well as ketone bodies, are increased. In adipose tissue catecholamines activate fat-mobilizing lipase activity presumably by enhancing the formation of cyclic AMP, and this causes lipolysis and FFA mobilization. We have the following temporal relationships between the effects of catecholamines and lipid metabolism: what we first observe is increased plasma levels of FFA followed by increased levels of triglyceride in the liver, and later on we have increased lipoprotein levels in the plasma. It is likely that of these events, the primary mechanisms are those occurring in adipose tissue leading to the increased mobilization of FFA and increased turnover of plasma FFA. Consequent upon the increased mobilization of FFA, there is an increased uptake of FFA into all tissues except the central nervous system, and this leads to increased amounts of triglycerides in the tissues; this permits the liver to enhance its secretion of triglyceride-rich lipoproteins into the plasma, and so we observe hypertriglyceridaemia.

\section{(b) Some physiological studies in dogs}

After this review, I will refer to one study we have done in anaesthetized dogs and later on some studies we have done in human subjects. We made a $24-\mathrm{hr}$ study in a group of control dogs and a group of dogs infused with noradrenaline, $1 \mu \mathrm{g} / \mathrm{kg} / \mathrm{min}$. The response seen in the latter is an immediate rise in plasma FFA, to reach about six times the normal level; this was followed by a gradual development of fatty livers. The triglyceride content of the liver increased about twenty to twenty-five times, and at this stage when you cut through them, the livers look like Danish butter. This was later on followed by a rise in plasma triglyceride levels, so you have the following time sequence: increase in FFA, increase in liver triglyceride, increase in plasma triglyceride. This plasma triglyceride increase was confined to the very low-density (pre- $\beta$ ) lipoprotein fraction. 
However, it is not only the liver which shows fatty infiltration; there is also a considerable fat infiltration in skeletal muscle during this excessive FFA mobilization. Interestingly, it is only the red fibres that get the fat infiltration; the white fibres do not. In addition, the myocardium develops fatty infiltration, and on electron microscopy you can see that the fat droplets accumulate very close to the mitochondria. And now in man-let us consider the normal FFA-triglyceride cycle with a normal rate of mobilization of FFA. When we stimulate this by catecholamines, we get exactly what we have seen in these dogs-a high plasma FFA level, tremendous increases in tissue triglyceride levels and eventually hypertriglyceridaemia.

\section{(c) Some physiological studies in man}

We are, of course, in our daily life exposed to several types of conditions which increase the activity of the sympathetic nervous system. I will show you two examples: one was a study we did in thirty normal subjects divided into three groups-one was a control group, a second group was subjected to standardized emotional stress for $2 \mathrm{hr}$, and the third group was also subjected to standardized emotional stress, but in addition was given nicotinic acid. This standardized stress procedure resulted in significant increases in the urinary output of both adrenaline and noradrenaline. The FFA levels in the controls remain unchanged; they increased significantly in the stressed group in response to this $2-\mathrm{hr}$ period of emotional stress, but there were no pronounced changes in the group given nicotinic acid-that is to say the increase was blocked by nicotinic acid. And interestingly enough it was possible, within the short period of 2-4 hr, to induce a very significant rise in plasma triglyceride levels. This rise was replaced by a fall in the group given nicotinic acid (Carlson, Levi \& Oro, 1968).

Another exposure we often have which may stimulate the activity of the sympathetic nervous system is coffee drinking, and I will briefly refer to our studies made in a group of about 100 students who were allocated into three groups: one group having caffeine-free coffee and two groups having caffeinecontaining coffee. In the group who consumed the caffeine-containing coffee the urinary excretion of noradrenaline and adrenaline increased significantly. Also this coffee drinking had a significant effect on the performance of the subjects on a standard test of intellectual agility. A rating of the number of mistakes remained unchanged during the study in the group having caffeine-free coffee, but in the two groups having caffeine in the coffee there was a significant improvement. So the caffeine had effects on performance as well as on secretion of catecholamines, but also unfortunately on plasma lipids.
Under the conditions of the study all groups were tested after a dinner. In the group having caffeinefree coffee there was a significant fall in plasma triglyceride. This fall was not found in the groups getting the caffeine; similarly, cholesterol levels fell in the group given the caffeine-free coffee, but rose in those consuming caffeine. This applies, of course, to Swedish coffee!

PROF. BoOTh: Well, thank you very much indeed, Professor Carlson-it was extremely interesting.

QUESTION: How do you standardize your emotional stress procedure?

Prof. CARLSON: The subjects were given a basket filled with steel balls of varying size and were asked to sort them out according to size. In addition, there was a considerable variation in the intensity of noise and of light from darkness to very bright light. All this was standardized; and we also hired an actor who, at given points, administered a sort of standardized criticism to the subjects telling them 'you really do not perform very well.' I have been exposed myself and it is really stressful!!

Question: How do you think, Professor Carlson, epinephrine release is stimulated? Is it a direct action on nerve-endings or is it mediated through the adrenal release of glucocorticoid?

Prof. Carlson: I do not think that glucocorticoids are involved here; I think it is a direct effect. Whether or not it is a direct effect on adrenal catecholamine release or comes from the sympathetic nerve-endings within adipose tissue I am not certain. There are sympathetic nerve-endings in adipose tissue also.

DR Dowling: May we ask you about the circulating lipids and the tissue levels of lipids in patients with phaeochromocytoma who have excessively high circulating catecholamine levels?

Prof. Carlson: Published evidence (Carlson et al., 1965) indicates that they certainly have elevated free fatty acid levels. I do not think that any one has looked into the tissue lipid levels of such patients. The situation is, of course, a bit more complicated than in our physiological studies because these patients are exposed to very much higher levels of catecholamines. They might, of course, get adapted to the mechanism.

Prof. BoOTH: Your studies are acute experiments, of course?

Prof. CARLSON: Yes, these are acute experiments.

QuEstion: Is the effect of adrenaline or noradrenaline working through alpha- or beta-sympathetic receptors on the adipose tissue?

Prof. Carlson: On adipose tissue the major effect is the stimulatory effect on the beta-receptors. A recent finding is that there might also be inhibitory effects on the alpha-receptors. Also we have the question of the blood flow in adipose tissue and both 
alpha- and beta-receptor mechanism might be involved with regard to regulation of blood flow.

DR KRIKLER: One would expect the beta-blockers would reduce this mobilizing effect.

Prof. Carlson: Yes, they do.

DR KRIKLER: Have you found that they do reduce this in acute and chronic experiments?

Prof. Carlson: We have not looked into that.

DR KRIKLER: Can I just mention that we have tried the effect of different beta-blockers in patients with elevated lipid levels, both cholesterol and triglycerides. We have compared two beta-blockers that also have some inherent sympathomimetic action, with propranolol, and we have seen no change whatsoever from control periods, after 2-week periods on treatment with accepted doses (Lloyd-Mostyn \& Krikler, 1970).

Prof. Carlson: Did you measure plasma FFA to be sure that FFA mobilization had been blocked?

DR KRIKLER: No we did not. We just measured the cholesterol and triglycerides.

PROF. CARLSON: It would be of interest, of course, to know the FFA.

PROF. BOOTH: Well, thank you very much indeed, Professor Carlson, for your extremely interesting presentation. Now Dr Krikler.

\section{Thyroid hormones and lipid metabolism}

DR KRIKLER: Unlike the two previous speakers, I have no original observations to report in the course of this review, apart from comments on the possible clinical usefulness of thyroid hormones in the management of hyperlipidaemia.

\section{(a) Mechanism of hypercholesterolaemia in myxoe- dema}

We all know that in hypothyroidism there is commonly an elevation of the serum cholesterol which is rapidly reduced after the administration of thyroid hormones. This can be used as supportive evidence for the presence of hypothyroidism. In the case of hyperthyroidism there is a tendency for the serum cholesterol levels to be subnormal but they are not usually so low as to be of diagnostic importance save under circumstances where subsequent antithyroid treatment produces an increase in the cholesterol level towards a more normal value. And it was this observation that gave rise to interest years ago in the usefulness of thyroid hormones in decreasing the serum cholesterol therapeutically, i.e. under circumstances other than hypothyroidism.

What is the mechanism whereby the serum cholesterol rise is brought about in hypothyroidism? The incorporation of ${ }^{14} \mathrm{C}$-labelled acetate into cholesterol is decreased, but there is an addition, a more extensive decrease in the catabolism of cholesterol in hypothyroidism; this has been shown by the slow rate of disappearance of radio-active cholesterol from the circulation which can be increased by the $c$ administration of thyroid hormone. In patients with hypothyroidism the decrease in catabolism is a more $\stackrel{?}{?}$ important factor than the decrease in the synthesis of $\bar{C}$ cholesterol; hence the hypercholesterolaemia that so regularly obtains. Now Miettinen (1968) has shown through some very elegant experiments the mechanism whereby thyroid hormones increase the catabolism of cholesterol: he gave tritium-labelled cholesterol intravenously to hypothyroid patients several weeks prior to starting administration of thyroid hormones. He confirmed that the serum cholesterol level was steady. He followed the fall in specific activity of the labelled material in the patient's plasma. After a period of observation varying between 4 and 6 weeks, he administered thyroid hormones in different combinations. This caused a very rapid fall in the serum cholesterol; in one case the level fell $\dot{0}$ from approximately 700 to just over $300 \mathrm{mg} / 100 \mathrm{ml} \sigma$ in 18 days. There was no further drop in the serum $ᄋ$ cholesterol nor was there any escape mechanism, i.e. the cholesterol remained down, approaching but not quite reaching a normal value. The mechanism of this fall in plasma cholesterol was an increase in the faecal excretion of endogenous neutral sterols There was a smaller and less constant increase io faecal bile acids (which are derived from cholesterol

(b) Clinical use of thyroid hormones to reduce serum cholesterol levels

I shall now turn to the therapeutic use of thyroid hormones in correcting hypercholesterolaemia. Laevothyroxine is unsuitable in patients suffering from coronary artery disease; an increase in the demands on the myocardium may be deleterious to those already suffering from coronary artery disease whether overt or latent, by precipitating arrhythmias or symptoms of angina, and it may in fact produce cardiac infarction. For this reason $L$-thyroxine itself is rarely used, certainly in recent years, in the treatment of hypercholesterolaemia. Now D-thyroxine was introduced some years ago, and I will consider why it may be safer and possibly have a greater utility in the treatment of this particular disorder. The concentration of D-thyroxine is more evenly distributed than L-thyroxine throughout the tissues (Tapley et al., 1959). There is, however, reason to $\sigma$ believe that this is not necessarily the whole story; $N$ some D-thyroxine can be incorporated in the myo- N cardium and could therefore produce deleterious $O$ effects; also it has been shown to increase the contractility of the myocardium in the cat. It is presumably because of the greater concentration of $\stackrel{\Phi}{\rightarrow}$ D- thyroxine in the liver and kidneys that it is metabolized more rapidly than is L-thyroxine; its half-life in the body is approximately $14 \mathrm{hr}$, as compared 
with $\mathbf{4 2} \mathrm{hr}$ for L-thyroxine. Its hypocholesterolaemic effect is nowhere near as great as that of L-thyroxine but it has proportionately a lower calorigenic effect; to put it another way, of all the thyroid hormones tried therapeutically it has the least calorigenic effect for a given hypocholesterolaemic effect.

D-Thyroxine is not necessarily completely safe. There have been some cases where angina has been produced. We have therefore embarked upon a trial using propranolol in addition to D-thyroxine. We do not have the full results yet, but we have found that our patients have tolerated this well, none having developed angina or other effects due to increased metabolism. We thus have reason to believe that the safety and thus effectiveness of $D$-thyroxine may be augmented by the concomitant use of propranolol. Perhaps in more severe cases the addition of other substances like cholestyramine may be of further help.

PROF. Booth: Thank you very much indeed.

Can I just clarify one point: You in fact are using D-thyroxine in patients with hypercholesterolaemia?

DR KRIKLER: Patients with hypercholesterolaemia due to hyperbetalipoproteinaemia, and some with ischaemic heart disease; but we use it only in conjunction with propranolol.

Prof. Booth: Well, I am sure people have comments to make on this.

DR Joplin: What dose, Dr Krikler?

DR KRIKLER: We started with a low dose of Dthyroxine, 2-4 mg a day, and increased after 2-4 weeks to $8 \mathrm{mg}$ a day, covered by propranolol, 20-40 $\mathrm{mg}$ three times daily.

DR THOMPSON: I was interested in your mention of the fact that an increased faecal excretion of neutral sterols occurs in patients treated with thyroid; it has been shown that if you give thyroid to rats, or if you have a patient with thyrotoxicosis, you can sometimes demonstrate malabsorption of dietary fat (Middleton \& Thompson, 1969), and it seemed to me possible that this could explain the increased excretion of neutral sterols. Do you know of any studies where the sterol excretion has been correlated with faecal fat?

DR KRIKLER: No.

DR THOMPSON: It is still possible that gastrointestinal hypermotility could give rise to relative malabsorption of both endogenous and dietary cholesterol. I just put this forward as a possible mechanism.

DR LEWIS: The patients we have looked at have not shown any consistent change in weight, and I am inclined to agree that increased faecal excretion of cholesterol and its products is a metabolic as opposed to an absorptive effect. Miettinen's work involved giving thyroxine as replacement therapy to hypothyroid patients; he was not inducing hyperthyroidism.
DR ElKeles: Would you not consider it dangerous to use two drugs which both affect myocardial metabolism in patients whose coronary supply may be suspect, in order to lower serum cholesterol, when other forms of treatment are available?

DR KRIKLER: Well, other methods do not always work that well and may have their own side-effects, and what we are doing is to see whether this combination removes any slight risk from the use of D-thyroxine, which others have used by itself without ill-effect (Council of Drugs, 1969). Boyd and Oliver (1960) thought that D-thyroxine was least likely to upset patients with coronary heart disease. We have not given it to patients who have severe angina; in our trial none of our twenty-four patients have developed angina or have had pre-existing angina aggravated during three months of treatment.

Prof. Booth: Professor Carlson, would you like to comment on this?

Prof. Carlson: Well, in 1960, we had five patients with severe hyper-betalipoproteinaemia and angina, and we gave them a very high dose of iodine-131 to make them hypothyroid, and then gave D-thyroxine, and we think that perhaps it had some useful effect; we tried to get a lower cholesterol level by substitution with a dose of D-thyroxine giving a normal basal metabolic rate. It was in fact quite possible to fully substitute them, when they had become hypothyroid, with D-thyroxine only. Now, there is one point here: if one needs a substitution dose of about $10 \mathrm{mg} /$ day of $\mathrm{D}$-thyroxine, a contamination of the D-thyroxine preparation with L-thyroxine of only $1 \%$, gives you $0.1 \mathrm{mg}$ of L-thyroxine, so I would like to ask you how pure your D-thyroxine is? We could never get $D$-thyroxine which was purer than $99 \%$.

DR KRIKLER: We were aware of this and I know that at the time that the original work was done a fair amount of the metabolic activity could be attributed to L-thyroxine contamination. We are, however, assured that this has been dealt with and our suppliers claim almost complete purity; in fact, by their analytical techniques, they state that they are unable to demonstrate any L-thyroxine at all.

DR Dowling: I wonder if I could just come back to Dr Thompson's question: I am intrigued by the mechanism whereby the D-thyroxine is having an effect on cholesterol metabolism, reducing the circulating levels of serum cholesterol and also promoting the faecal excretion of cholesterol. Is this in the absence of an associated increase in faecal excretion of bile acids?

DR KRIKLER: No, these increases are not just cholesterol itself; there was also a smaller and less consistent rise in bile acids.

DR DowlING: This was Miettinen's study; I just wondered if he was using a formula diet, because I think his studies with Dr Ahrens are a little bit at 
variance with most people's findings in relation to, for example, the effects of diet on the excretion of neutral sterols and bile acids. They, for example, claim that they had no increase in faecal excretion of bile acids on changing from saturated to unsaturated fat, whereas most people do observe this effect. Was Miettinen's thyroid study in fact carried out on a formula diet?

DR KRIKLER: Well, he was not using a formula diet, but a diet which was rigidly controlled and standardized from day-to-day, the constituents of which were known; and he was using either betasitosterol or chromic oxide as markers of faecal flow.

Prof. Booth: Any other queries?

QUESTION: Why is it that the cholesterol increases so much in primary hypothyroidism but not in hypothyroidism secondary to pituitary disease?

Prof. Russell Fraser: First of all, let us say that most patients with secondary hypothyroidism do show a rise in serum cholesterol, but if you have twenty hypopituitary patients to contrast with twenty primary hypothyroid patients the mean levels are different, as several studies have shown. When you ask the question of why the discrepancy, I do not telieve anybody knows the answer.

DR JOPLIN: On the other hand, if you maintain such hypopituitary patients alive on corticosteroids and permit them to have untreated myxoedema their cholesterols can go up to $600 \mathrm{mg} / 100 \mathrm{ml}$ in a matter of a few months, so there may be no fundamental difference at all. It may be just that totally untreated hypopituitarism is a self-limiting disease; they cannot survive long enough to develop severe myxoedema.

Prof. Booth: Professor Fraser, did you have another point?

Prof. Russell Fraser: I do not doubt that, with an equivalent degree of failure of the thyroid, there is a slightly lesser raising of cholesterol in hypopituitarism. You can, of course, get quite high levels of cholesterol in hypopituitarism.

DR LEWIS: One further brief point about the results of Dr Krikler's and my study with dextrothyroxine; it is rather fascinating to note that some patients responded dramatically well to this therapy and others not at all.

PROF. BOOTH: You mean chemically or in terms of symptoms?

DR LEWIS: The fall in serum cholesterol is variable in patients with identical hyperbetalipoproteinaemia. One possible inference might be that such patients are metabolically heterogeneous.

Prof. BooTh: Right, I think we will have to move on now. Dr Thompson.

\section{Sex hormones and lipid metabolism}

DR ThOMPSON: My recently acquired knowledge of the biochemistry of sex has suggested to me that this field is full of rather unexpected and inexplicable findings. Now, as far as sex hormones and lipids are concerned, I will try as far as I can to classify those points that seem to be clear-cut and not confuse you too much with those that are not. I am going to talk about two main aspects of this subject: firstly about the differences in males and females in terms of serum lipid levels, and its possible relationship to ischaemic heart disease; and secondly, the effect of oral contraceptives on serum lipids.

(a) Effect of administration of individual sex hormones

As far as serum lipids are concerned, oestrogens have been shown to lower low-density lipoproteins (LDL) and to increase very low-density (VLDL) and high-density lipoproteins (HDL). As a result, if you give oestrogens you find that there is a fall in the serum cholesterol (a LDL) and often a rise in the triglyceride (a VLDL) and phospholipid fraction. In the past this has often been expressed by saying that the cholesterol : phospholipid ratio has fallen. Androgens have the opposite effect on each of these lipoproteins, so that you will find a rise in lowdensity lipoproteins and a fall in the other two classes. As far as progestagens are concerned, they do noto seem to have any clear-cut effect on serum lipids buto it has been suggested that certain of them are somewhat androgenic in structure and may have an antioestrogenic effect.

(b) Serum lipid and lipoprotein levels in males and females

Now, following on what I have just said, we should be able to show differences between the serum lipoprotein concentrations in males and females. As far as low-density lipoproteins are concerned, they are higher in men than in women, and the reverse applies to high-density lipoproteins. Now, initially I said that if you give oestrogens you raise the level of very low-density lipoprotein, and here is one of those anomalies, that in spite of this, males have higher levels of very low-density lipoprotein than females.

Following on these differences in lipoprotein concentrations one should be able to show differences between the sexes in serum lipid concentrations. To take serum cholesterol, first of all, data presented at a recent symposium on sex hormones and lipids by Bierman (1969), has shown, and many other authors have confirmed this, that up to the age of 45 men have a higher serum cholesterol than do women. However, after this age, and certainly beyond the age of 55 , you find the reverse and men have a lower serum cholesterol than do women. Now there are two possible explanations for this, and I do not know that either of them is necessarily the correct or sole one, but one is that after the menopause the 
female supply of oestrogen runs out, its protective effect is lost and the serum cholesterol rises. There are a number of people who would dispute this and say if that was so, one would expect to find a curve which was initially flatter and which then rose sharply at 55, instead of the rather smooth and linear increase that occurs (Furman, Alaupovic \& Howard, 1967). An alternative explanation is that men with high serum cholesterols have tended to be eliminated during this period and that is why you do not find them present after the age of 55 .

As far as serum triglyceride levels are concerned, there is again a difference between the sexes, which is similar to cholesterol, in that males have higher triglyceride levels than do females up to the age of 55, whereas after that age they tend to run in parallel.

As far as the difference in mortality between males and females below the age of 55 is concerned, it seems likely that this is in some way related to the higher serum cholesterol and triglyceride levels that occur in males. However, there may be some dispute about this relationship because in America there is a $5: 1$ ratio of male : female incidence of ischaemic heart disease in pre-menopausal age-groups, whereas in Japan the ratio is only about $1: 1$. Since women are women the whole world over it may be that dietary factors are equally as important as hormonal ones in determining the difference between males and females in respect of ischaemic heart disease.

Whatever the explanation for the difference between the sexes as far as ischaemic heart disease is concerned, there is undoubtedly good evidence that if you give oestrogens to men you can lower their serum cholesterol. This is clear from the work of Oliver \& Boyd (1961), who took a group of patients who had had a myocardial infarct and divided them into two groups. One group acted as controls, whereas an equal number of similar patients were treated with oestrogens and showed quite a satisfactory and maintained fall in the serum cholesterol levels over a 5-year period. When the oestrogens were stopped they had a rebound in serum cholesterol levels. The fall in the cholesterol : phospholipid ratio which occurred suggested that the effect of oestrogens was mainly by way of its action in lowering the low-density lipoprotein fraction. However, in spite of these changes in serum lipids, there was no increase in the number of survivors in the oestrogen-treated group as compared to the control group, so that oestrogen had not in any way influenced mortality. The use of oestrogens for lowering serum cholesterol in men has now gone out of favour because of the high incidence of feminising sideeffects.

\section{(c) Effect of oral contraceptives}

Now I should like to turn to the topic of the effect of oestrogenic contraceptives on serum lipids. There is some data, again from the work of Bierman's group, which shows quite clearly that in some women on oestrogenic contraceptives there is quite a marked increase in the serum triglyceride levels (Hazzard $e t$ $a l, 1969)$. This is presumably in part due to the decrease in post-heparin lipolytic activity (PHLA) which these authors found in their patients. In addition to this decrease in PHLA they also showed an increase in immunoreactive insulin levels in these patients. Now, as far as the mechanism of this hypertriglyceridaemia which can be induced by some oral contraceptives is concerned, the authors proposed that there were two possible mechanisms - first of all that oestrogens were antagonizing insulin, the concomitant increase in secretion of insulin and synthesis of endogenous triglyceride leading to an increased flux of triglyceride; and secondly they postulated that oral contraceptives were antagonizing lipoprotein lipase and were thus decreasing the removal of triglyceride from the circulation. The actual rise in triglyceride levels was really quite substantial, something in the region of $40-50 \mathrm{mg} / 100 \mathrm{ml}$. Finally, to conclude, I should like to draw your attention to the work of Glueck and his colleagues (1969b), who showed that the hypertriglyceridaemia and decreased PHLA found in certain patients with Type 5 hyperlipoproteinaemia could be restored to normal by treatment with a progestagen. This is a very interesting observation and led these authors to speculate that a suitable combination of a progestagen and an oestrogen might be able to prevent the rise in serum triglycerides that may occur in patients receiving oestrogens alone as oral contraceptives.

Prof. Booth: Well, thank you very much Dr Thompson.

Prof. Carlson: I think we in the lipid field must ask for apologies because it seems that people have to forgo many things; we have just talked about the undesirable effects of coffee, and we are now talking about the effects of the contraceptive pill, which are not very favourable. We too have made a study on oestrogen-containing contraceptive pills, the results of which are very similar to those obtained by $\mathrm{Dr}$ Bierman.

Prof. Booth: You are suggesting sex is out, too, are you?

Prof. CARLson: We gave an oestrogen-containing contraceptive for 2 months to twelve healthy young women; there was an increase in triglycerides of $200 \%$ above the basal, and the interesting thing is that this increase persisted for quite some time after stopping taking the pill. It is still significant even 1 month after stopping. There was also a small rise in the cholesterol values, and still at 6 weeks after stopping taking the pill there is seen a significant increase above basal. We could in this study confirm what 
has been told by Bierman and his co-workers that there was about $50 \%$ reduction in lipoprotein lipase level (PHLA) but interestingly enough, when we looked at the fat tolerance test to assess the clearing of plasma triglyceride this was unchanged, so we would rather favour the idea that the increase in triglyceride reflects increased hepatic synthesis of very low-density lipoprotein. We also analysed lipoprotein fractions in these young women, and saw that the increase in lipoprotein was confined to the very low-density lipoproteins and to the high-density lipoproteins. It is worthwhile to know that the effect persists.

DR Lewis: Could I ask Professor Carlson what his view is on a reduction of PHLA of this sort of magnitude (say $50 \%$ ). This sort of reduction is described in a large number of clinical situations and experimental ones, and I wonder whether it is likely to be rate-limiting to triglyceride removal.

Prof. Carlson: Well, I think this is a very important question and certainly back in the 1950 s we hoped very much that one should have clinically useful information from measuring PHLA. As yet we can only with confidence say that we have interesting clinical information when there is complete absence of post-heparin lipolytic activity. What the reduced levels mean I am not sure. Perhaps you have another viewpoint, Dr Robinson.

DR RoBINSON: I agree with you entirely.

COMMENT: If oral contraceptives have to be avoided, so too is pregnancy, because you get very high cholesterols as well as triglycerides in pregnancy, and this does not seem to do much harm to the women.

Dr ANDERSON: The androgens push up the serum cholesterol level and yet I understand you sometimes give oxymetholone, which is a potent synthetic androgen, with the aim of bringing down the serum cholesterol. What was the reason for this?

DR LEWIS: There is published evidence that oxymetholone reduces cholesterol and triglyceride levels rather effectively. The mechanisms involved are quite unknown. The drug is chiefly anabolic in its effects, rather than androgenic. Our own experience of some twenty cases is that oxymetholone is certainly valuable in endogenous hypertriglyceridaemia. We used a dose of only $5 \mathrm{mg}$ twice or three times daily.

DR THOMPSON: The effect of androgens is predominantly on low-density lipoprotein which is not a particular carrier of triglycerides, so $I$ think it would be difficult to see how this could be so.

Prof. Booth: Well, I think we ought to go and have practical exercise on effect of diet on our lipid handling. Thank you all.

\footnotetext{
Acknowledgment

This conference was recorded and edited by Dr G. F. Joplin.
}

\section{References}

Abrams, M.E., Jarrett, R.J., Keen, H., Boyns, D.R. \& Crossland, N. (1969) Oral glucose tolerance and related factors in a normal population sample. British Medical Journal, 1, 599.

Albrink, M.J., Lavietes, P.H. \& MaN, E.B. (1963) Vascular disease and serum lipids in diabetes mellitus. Annals of Internal Medicine, 58, 305.

BAKER, N. (1968) Hepatic triglyceride secretion in relation to lipogenesis and free fatty acid mobilization in fasted and glucose-refed rats. Journal of Lipid Research, 9, 1.

Basso, L.V. \& HAVEL, R.J. (1970) Hepatic metabolism of free fatty acids in normal and diabetic dogs. Journal of Clinical Investigation, 49, 537.

BIERMAN, E.I. (1969) Conference on Metabolic Effects of Gonadal Hormones and Contraceptive Steroids (Ed. by Salhanick, Kipnis and van de Weile), p. 207. New York.

BOYD, G.S. \& Oliver, M.F. (1960) The effect of certain thyroxine analogues on the serum lipids in human subjects. Journal of Endocrinology, 21, 33.

Carlson, L.A., Boberg, J. \& Hogstedt, B. (1965) Some physiological and clinical implications of lipid mobilization from adipose tissue. In: Handbook of Physiology-Adipose Tissue (Ed. by A. E. Renold and G. F. Cahill), p. 625. Americal Physiological Society.

Carlson, L.A., Levi, L. \& Oro, L. (1968) Plasma lipids and urinary excretion of catecholamines in man during experimentally induced emotional stress, and their modification by nicotinic acid. Journal of Clinical Investigation, 47, 1795.

CouncIL ON DRUGS (1969) Evaluation of a hypercholesterolemic agent. Journal of American Medical Association, 208 1014.

Dole, V.P. (1956) A relation between non-esterified fatt acids in plasma and the metabolism of glucose. Journal Clinical Investigation, 35, 150.

Furman, R.H., Alaupovic, P. \& Howard, R.P. (1967) Hormones and lipoproteins. Progress in Biochemistry Pharmacology, 2, 215.

GlueCK, C.J., LeVY, R.I. \& Fredrickson, D.S. (1969a) Immunoreactive insulin, glucose tolerance, and carbohydrate inducibility in types II, III, IV and V hyperlipoproteinaemia. Diabetes, 18, 739.

GlueCK, C.J., Brown, W.V., Levy, R.I., Greten, H. \& FredRICKSON, D.S. (1969b) Amelioration of hypertriglyceridaemia by progestational drugs in familial type-V hyperlipoproteinaemia. Lancet, i, 1290.

GoRDon, R.S. \& Cherkes, A. (1956) Unesterified fatty acid in human blood plasma. Journal of Clinical Investigation, 35, 206.

Greenough, W.B., Crespin, S.R. \& Steinberg, D. (1967) Hypoglycaemia and hyperinsulinaemia in response to raised free-fatty acid levels. Diabetes, 17, 244.

HAFT, D.E. (1968) Studies of the metabolism of isolated livers of normal and alloxan-diabetic rats perfused with insulin. Diabetes, 17, 244.

Hazzard, W.R., Spiger, M.J., Bagdade, J.D. \& Bierman, N E.L. (1969) Studies on the mechanism of increased plasma $\sigma$ triglyceride levels induced by oral contraceptives. New N England Journal of Medicine, 280, 471.

Jackson, R.A., Peters, N., Advani, U., Perry, G., Rogers, J. \& Pilkington, T.R.E. (1970) Peripheral glucose utilization in young men with myocardial infarction. Clinical $O$ Science, 38, 32P.

JONES, D.P. \& ARKY, R.A. (1965) Effects of insulin on triglyceride and free fatty acid metabolism in man. Metabolism, 14, 1287.

Lloyd-Mostyn, R.H. \& Krikler, D.M. (1970) Raised serum-lipids unaffected by beta-blockade. Lancet, ii, 1040 . 
Middleton, W.R.J. \& Thompson, G.R. (1969) The mechanism of steatorrhea in induced hyperthyroidism in the rat. Journal of Laboratory Clinical Medicine, 74, 19.

MietTinen, T.A. (1968) Mechanism of serum cholesterol reduction by thyroid hormones in hypothyroidism. Journal of Laboratory Clinical Medicine, 71, 537.

OLIVER, M.F. \& BOYD, G.S. (1961) Influence of reduction of serum lipids on prognosis of coronary heart disease. Lancet, ii, 499.

Randle, P.J., Garland, P.B., Hales, C.N. \& Newsholme, E.A. (1963) The glucose fatty-acid cycle, its role in insulin sensitivity and the metabolic disturbances of diabetes mellitus. Lancet, i, 785.

Reaven, G.M., Lerner, R.O., Stern, M.P. \& Farquhar, J.W. (1967) Role of insulin in endogenous hypertriglyceridaemia. Journal of Clinical Investigation, 46, 1756.
Renold, A.E., Hastings, A.B., Nesbett, F.B. \& Ashmore (1955) Studies on carbohydrate metabolism in rat liver slices. Journal of Biological Chemistry, 213, 135.

Stout, R.W. \& Vallance-OWen, J. (1969) Insulin and atheroma. Lancet, i, 1078.

TAPleY, D.F., Dandoff, F.F., Hatfield, W.B. \& Ross, J.E. (1959) Physiological disposition of D- and L-thyroxine in the rat. American Journal of Physiology, 197, 1021.

Tzagournis, M., Chiles, R., RYAN, J.M. \& SkIllinous, T.G. (1968) Interrelationships of hyperinsulinism and hypertriglyceridaemia in young patients with coronary heart disease. Circulation, 38, 1156.

van Harken, D.R., Brown, T.O. \& Heimberg, M. (1967) Hepatic lipid metabolism in experimental diabetes. III. Synthesis and utilization of triglycerides. Lipids, 2, 231.

WADSTROM, L.B. (1957) Lipolytic effect of the injection of adrenaline on fat depots. Nature, 179, 259. 